INPLASY

PROTOCOL

To cite: Yao et al. Joint mobilization for frozen shoulder: A protocol for systematic review and meta analysis. Inplasy protocol 202210075. doi:

10.37766/inplasy2022.1.0075

Received: 16 January 2022

Published: 16 January 2022

Corresponding author:

Qiangqiang Shang

470396919@qq.com

Author Affiliation:

Department of Tuina, the

Affiliated Hospital to

Changchun University of

Chinese Medicine, China.

Support: JJKH20220870KJ.

Review Stage at time of this submission: Preliminary searches.

Conflicts of interest:

None declared.

\section{Joint mobilization for frozen shoulder: A protocol for systematic review and meta analysis}

\author{
Yao, J1; Liu, C2; Pang, T3; Li, J4; Wang, Y5; Shang, Q6.
}

Review question / Objective: Population: Patients with frozen shoulder; Intervention: Joint mobilization; Comparison: Rehabilitation therapy; Outcome: joint range of motion (ROM) and Japanese Orthopedic Association (JOA) score.

Information sources: Related studies in the following databases will be searched from inception to December 30, 2021: Medicine, PubMed, Embase, Web of Science, Cochrane Library, China National Knowledge Infrastructure, Wan-Fang Database, Chongqing VIP Chinese Science and Technology Periodicaols Database and China Biomedical Database.

INPLASY registration number: This protocol was registered with the International Platform of Registered Systematic Review and Meta-Analysis Protocols (INPLASY) on 16 January 2022 and was last updated on 16 January 2022 (registration number INPLASY202210075).

\section{INTRODUCTION}

Review question / Objective: Population: Patients with frozen shoulder; Intervention: Joint mobilization; Comparison: Rehabilitation therapy; Outcome: joint range of motion (ROM) and Japanese Orthopedic Association (JOA) score.
Condition being studied: Frozen shoulder is a clinical syndrome characterized by shoulder pain and dysfunctional movement due to soft tissue lesions around the shoulder joint. The literature suggests that the global prevalence of frozen shoulder is between $2 \%$ and $5 \%$. Most people who develop frozen shoulder are between $\mathbf{4 0}$ and 60 years of age, except for secondary traumatic frozen shoulder, which is 
uncommon in patients over 70 years of age. The incidence in women is 1.6 of that in men. A variety of frozen syndromes exist clinically, with frozen shoulder being the most common "frozen" syndrome. The exact cause of frozen shoulder is unknown, but the disease is currently thought to be the result of a combination of synovial inflammation and joint capsule fibrosis. The clinical symptoms of the disease are mostly pain in the shoulder, with severe pain at night or even insomnia, and severe limitation of shoulder movement, especially abduction and external rotation, often in a state of "freezing, condensation and knotting", hence the name frozen shoulder. Current research has found that a large number of patients with frozen shoulder do not experience spontaneous resolution of their symptoms. The common non-surgical treatments include medication, physical therapy, exercise, manipulation under anesthesia, steroid injections or nerve blocks, which can temporarily relieve the symptoms, but the results are inaccurate, recurring, with side effects, long treatment intervals and poor patient compliance. It can restore normal joint gliding and improve symptoms within a short treatment period. Mulligan arthrodesis is highly valued by patients for its efficacy, ease of use, and excellent prognosis. Although many clinical studies have reported good efficacy of arthrodesis in the treatment of frozen shoulder, there is not sufficient clinical evidence. Therefore, this systematic review aims to evaluate the effectiveness and safety of arthrodesis for frozen shoulder and to provide a better basis for clinical decision making.

\section{METHODS}

Participant or population: All patients with frozen shoulder will be included without limitation of age, race, sex, economic level, and severity.

Intervention: The interventions in the experimental group included only joint mobilization. It mainly included different levels and types of joint mobilization.
Comparator: Interventions in the control group only included rehabilitation therapy.

Study designs to be included: Only randomized controlled trials (RCTs) will be included in this study.

Eligibility criteria: Nonrandomized controlled studies case reports, case series and reviews will not be included in this study.

Information sources: Related studies in the following databases will be searched from inception to December 30, 2021: Medicine, PubMed, Embase, Web of Science, Cochrane Library, China National Knowledge Infrastructure, Wan-Fang Database, Chongqing VIP Chinese Science and Technology Periodicaols Database and China Biomedical Database.

Main outcome(s): The primary outcome was joint range of motion (ROM) and Japanese Orthopedic Association (JOA) score.

Additional outcome(s): Secondary endpoints included Visual Analogue Scale (VAS) score, Activities of Daily Living (ADL) score.

Quality assessment / Risk of bias analysis: The quality of all RCTs will be evaluated with the Cochrane Collaboration tool. Two authors (Tingting Pang and Chang Liu) will independently conduct quality evaluations, and any controversy will be addressed by discussion with another author (Junjie Yao).

Strategy of data synthesis: The metaanalysis of data from included outcomes will be performed using the RevMan V.5.4.1 and we will choose a randomized or fixed effect model for data statistics according to the results of the heterogeneity test. The enumeration data were expressed as relative risk (RR), and the weight mean difference (WMD) was used as the measurement data; each effect amount was expressed in $95 \%$ confidence interval (Cl). The specific methods were as follows: If the heterogeneity was low $\left(I^{2}<50 \%\right.$, the fixed-effects model was used for data 
synthesis. If there is high heterogeneity $\left(I^{2}>\right.$ $50 \%$ ), the random-effects model will be used for data synthesis after excluding possible heterogeneity sources. The investigation methods included subgroup and sensitivity analyses. If data cannot be synthesized, we provide a descriptive analysis to solve this problem.

Subgroup analysis: If there was high heterogeneity $(12>50 \%)$ among the included studies, we conducted a subgroup analysis to analyze the sources of heterogeneity according to the following factors: age, sex, race, courses, sample sizes, different levels and types of joint mobilization, and other possible factors affecting the results.

Sensitivity analysis: To test the stability and reliability of the results of this study, we conducted a sensitivity analysis according to the following points: method quality, sample size, and missing data. After that, we will perform a data analysis again and compare the results. If there was no directional change after the sensitivity analysis, the results were stable.

Language: English and Chinese.

Country(ies) involved: China.

Keywords: Systematic review; Metaanalysis; Joint mobilization; Rehabilitation; Frozen shoulder.

Contributions of each author:

Author 1 - Junjie Yao.

Email: 2671032365@qq.com

Author 2 - Chang Liu.

Email: 1483230590@qq.com

Author 3 - Tingting Pang.

Email: 815483770@qq.com

Author 4 - Jiahui Li.

Email: 2394690171@qq.com

Author 5 - Yufeng Wang.

Email: wangchn@126.com

Author 6 - Qiangqiang Shang.

Email: 470396919@qq.com 